\title{
Expression of apical junction complex proteins in colorectal mucosa of miniature dachshunds with inflammatory colorectal polyps
}

\author{
Nozomu YOKOYAMA ${ }^{1)}$, Hiroshi OHTA ${ }^{1)}$, Yumiko KAGAWA ${ }^{2)}$, \\ Rommaneeya LEELA-ARPORN ${ }^{1)}$, Angkhana DERMLIM ${ }^{1}$, , Khoirun NISA ${ }^{1}$,

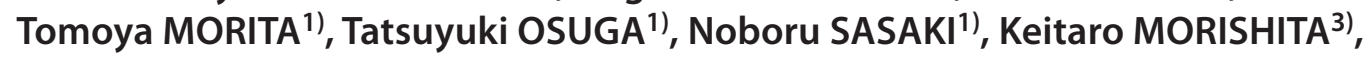 \\ Kensuke NAKAMURA ${ }^{3)}$ and Mitsuyoshi TAKIGUCHI ${ }^{1 *}$
1)Laboratory of Veterinary Internal Medicine, Department of Veterinary Clinical Sciences, Graduate School of Veterinary Medicine, Hokkaido University, Sapporo, Hokkaido 060-0818, Japan
2)Department of Diagnostic Pathology, Graduate School of Veterinary Medicine, Hokkaido University, Sapporo, Hokkaido 060-0818, Japan
${ }^{3)}$ Veterinary Teaching Hospital, Department of Veterinary Clinical Sciences, Graduate School of Veterinary \\ Medicine, Hokkaido University, Sapporo, Hokkaido 060-0818, Japan
}

\section{J. Vet. Med. Sci.}

79(3): 456-463, 2017

doi: 10.1292/jvms.16-0479

Received: 12 September 2016 Accepted: 29 December 2016 Published online in J-STAGE: 16 January 2017

\begin{abstract}
We examine the expression of tight junction and adherence junction proteins in the colorectal mucosa of miniature dachshunds (MDs) with inflammatory colorectal polyps (ICRPs). Colorectal mucosa samples were endoscopically obtained from 8 MDs with ICRPs and 8 control dogs for immunoblotting. Paraffin-embedded tissues of surgically resected inflamed lesions from another $5 \mathrm{MDs}$ with ICRPs and full-thickness colorectal specimens from 5 healthy beagles were obtained for immunohistochemistry. The expression patterns of claudin- $-1,-2,-3,-4,-5,-7$ and -8 , E-cadherin and $\beta$-catenin were analyzed in the non-inflamed mucosa and inflamed mucosa of ICRPs and colorectal mucosa of control dogs by immunoblotting. The localization of these proteins in the inflamed lesions was analyzed by immunohistochemistry. The expressions of each of claudin, E-cadherin and $\beta$-catenin were not significantly different between control dogs and noninflamed colonic mucosa from MDs with ICRPs. In contrast, only E-cadherin and $\beta$-catenin were detected in the inflamed lesions of MDs with ICRPs. By immunohistochemistry, claudin-2, $-3,-4,-5$ and $-7, E$-cadherin and $\beta$-catenin were expressed in the colorectal epithelium within the inflamed mucosa, but not in granulation tissue. Distributions of claudin-2,-3, -4, -5 , and -7 , E-cadherin and $\beta$-catenin in the colonic epithelium were not different between MDs with ICRPs and control dogs. These results indicated that no significant alteration was detected in several tight junction or adherence junction proteins expression in the colorectal epithelium of ICRPs.

KEY WORDS: apical junction complex proteins, colorectal mucosa, inflammatory colorectal polyps, miniature dachshund
\end{abstract}

Inflammatory colorectal polyps (ICRPs) in Miniature dachshunds (MDs) were recently recognized as a major cause of large bowel diarrhea in this dog breed in Japan [13]. ICRPs are characterized by the formation of multiple small polyps around the large bowel mucosa, almost invariably restricted to the descending colon and rectum, sometimes accompanied by space-occupying solitary large polyp formation. Histopathological findings of ICRPs are proliferative changes of epithelium without cellular atypia, increased goblet cells with dilated crypts, the infiltration of inflammatory cells (predominantly by neutrophils and macrophages) and proliferation of granulation tissue $[13,20]$. MDs with ICRPs show a good response to immunosuppressive drugs, such as prednisolone, cyclosporine and leflunomide $[6,13]$. Based on the existence of idiopathic inflammation and effectiveness of immunosuppressive therapy, ICRPs are thought to be a novel form of breed-specific canine inflammatory bowel disease (IBD) in MDs $[8,9,16]$.

Canine and human IBD is a group of disorders characterized by persistent or recurrent gastrointestinal signs and histologic evidence of intestinal inflammation [4]. The pathophysiology of canine and human IBD is not definitively known. However,

*Correspondence to: Takiguchi, M., Laboratory of Veterinary Internal Medicine, Graduate School of Veterinary Medicine, Hokkaido University, N18 W9, Sapporo, Hokkaido 060-0818, Japan.e-mail: mtaki@vetmed.hokudai.ac.jp

(02017 The Japanese Society of Veterinary Science

This is an open-access article distributed under the terms of the Creative Commons Attribution Non-Commercial No Derivatives (by-nc-nd) License. (CC-BY-NC-ND 4.0: https://creativecommons.org/licenses/by-nc-nd/4.0/) 


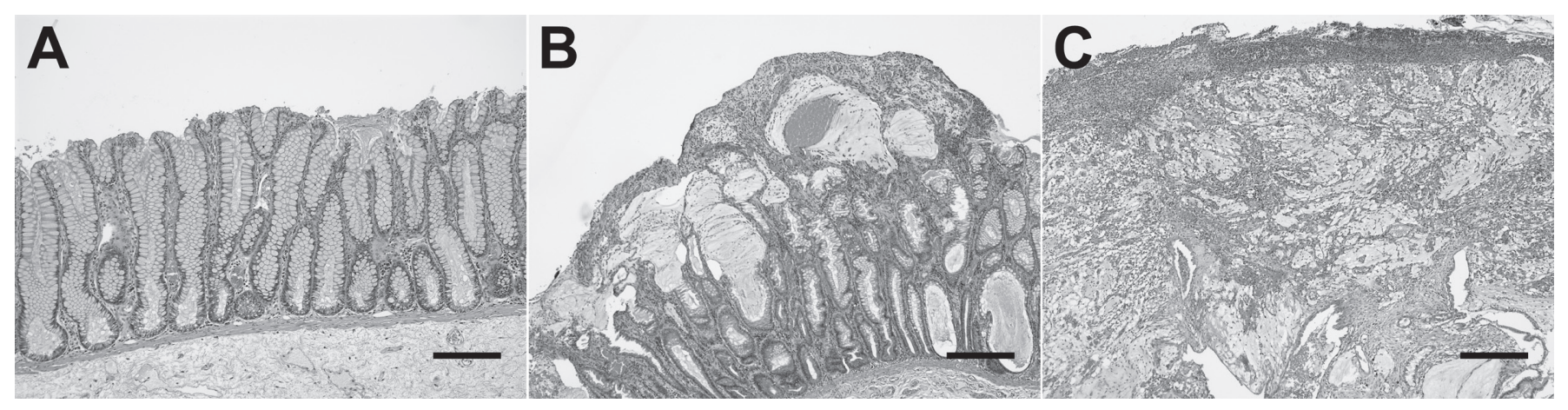

Fig. 1. Representative photomicrograph of the histologic section of an inflamed lesion of miniature dachshund (MD) with inflammatory colorectal polyps (ICRPs). Histopathological appearance of stage I (A), stage II (B) and stage III (C). (A) Stage I, thickened mucosa composed of hyperplastic goblet cells. (B) Stage II, granulation tissue formation in the apical region of the mucosa and dilated crypts containing abundant mucus. (C) Stage III, excessive production of granulation tissue, abundant neovascularization and marked inflammatory changes. H\&E stain; Bar=200 $\mu \mathrm{m}$ for $\mathrm{A}$ and $300 \mu \mathrm{m}$ for B and $\mathrm{C}$.

factors that may be important for the pathogenesis of IBD include changes in mucosal barrier functions and mucosal immune system dysfunction with a loss of tolerance to endogenous microflora or dietary antigens, resulting in chronic inflammation of the gastrointestinal tract [22].

The intestinal epithelial barrier is established by a single layer of epithelial cells, and the space between these cells is sealed by tight junctions (TJs) and adherence junctions (AJs), which regulate the permeability of the intestinal barrier. TJs are complex protein structures comprised of transmembrane proteins. A major component of TJ strands is the integral proteins, claudins. Claudins are transmembrane proteins, and there are 27 members of the claudin family [2]. Differential expressions and properties of claudins are believed to determine tissue-specific variations in electrical resistance and paracellular ionic selectivity among epithelia. AJs are composed of the transmembrane protein E-cadherin and the associated cytoplasmic proteins, the catenins; these are important in the formation of TJs [3]. Alterations in TJ and AJ protein expression and localization were reported in human and canine IBD [11]. In previous reports, increased expression of claudin-2 and decreased expression of claudin-3, $-4,-7$ and -8 were demonstrated in the colonic mucosa of human IBD $[1,23]$. In our previous study, E-cadherin expression was significantly decreased in the villus epithelium in duodenal mucosa samples obtained from dogs with IBD [15].

Previously, we determined the protein expression and distribution of several claudins, E-cadherin and $\beta$-catenin in the colonic mucosa of healthy beagles [14]. However, there is no information regarding the expression of these TJ and AJ proteins in the colorectal mucosa of MDs with ICRPs. The objective of this study was to determine the expression and distribution of various TJ proteins (claudin-1, -2, -3, -4, -5, -7 and -8) and AJ proteins (E-cadherin and $\beta$-catenin) in the colorectal mucosa obtained from MDs with ICRPs.

\section{MATERIALS AND METHODS}

\section{Animals}

For immunoblot analysis, eight MDs with ICRPs and 8 control dogs were used in this study. The 8 MDs with ICRPs had been referred to Hokkaido University Veterinary Teaching Hospital for the investigation of hematochezia, tenesmus and an increased frequency of defecation and diagnosed with ICRPs based on clinical and histopathological findings of a previous report [13, 20]. They consisted of 5 males ( 2 intact and 3 neutered) and 3 females ( 2 intact and 1 neutered), and the median age of these dogs was 10 years (range, 9 to 14 years). Written owner consent was obtained for the inclusion of dogs in this study. Control tissue samples were obtained from 8 healthy dogs in a research colony ( 4 beagles and 4 mixed-breed dogs). These healthy dogs consisted of 5 intact males and 3 intact females, and their median age was 2 years (range, 1 to 9 years). None of these dogs had clinical signs of weight loss for more than 1 year before sample collection. Hematologic, serum biochemical, fecal and abdominal ultrasonographic examinations were performed for all dogs.

For immunohistochemistry analysis, we used the paraffin-embedded tissues of large inflamed lesions collected from another 5 MDs with ICRPs. Rectal pull-through excision was performed at private veterinary hospitals, and large inflamed tissues were submitted to the Department of Diagnostic Pathology of Graduate School of Veterinary Medicine, Hokkaido University for histopathological examination. These $5 \mathrm{MDs}$ consisted of 2 neutered males and 3 females ( 1 intact and 2 neutered), and the median age of these dogs was 9 years (range, 8 to 11 years). Inflamed mucosal lesions were staged (I-III) according to criteria adapted from those described previously [20]. Lesions in which $>15,5-15$ and $<5 \%$ of the tissue consisted of epithelial cells were defined as stages I, II and III lesions, respectively (Fig. 1). As a control, full-thickness colorectal tissue samples were obtained from 5 healthy intact male beagles (median age, 0.6 years; range, 0.6-0.7 years), which were used in another unrelated study, at necropsy immediately after euthanasia by an intravenously administered overdose of pentobarbital. These dogs had no clinical signs of gastrointestinal disease and showed no abnormalities, as determined by hematologic, serum biochemical, fecal and abdominal 
ultrasonographic examinations. The samples were immediately put into $10 \%$ phosphate-buffered formalin and embedded in paraffin. These control biopsy specimens showed no abnormality based on World Small Animal Veterinary Association guidelines $[5]$.

All experimental procedures in this study were approved by the Animal Care and Use Committee and the Laboratory Animal Experimentation Committee, Graduate school of Veterinary Medicine, Hokkaido University (Accession number, 13-0142 and 14-0031, respectively).

\section{Tissue sample collection}

Colorectal mucosa biopsy samples were endoscopically obtained. Colonoscopy was performed during anesthesia with a flexible video endoscope (VQ-8143A, AVS, Tokyo, Japan), and multiple (6 to 8) mucosal biopsy samples of inflamed lesions of 8 MDs with ICRPs were collected using serrated biopsy forceps (FB-53Q-1, AVS). In addition, mucosal biopsy samples of the descending colonic mucosa without macroscopic lesions (non-inflamed mucosa) were also collected from these MDs with ICRPs. Control samples were also obtained from descending colonic mucosa of 8 healthy dogs in a research colony. Biopsy samples for histologic examination were fixed in neutral buffered $10 \%$ formalin and then embedded in paraffin wax. Biopsy samples were obtained from each dog for immunoblot analysis, and these samples were frozen and stored at $-80 \mathrm{C}^{\circ}$. Biopsy specimens from the descending colonic mucosa of 8 MDs with ICRPs and 8 healthy dogs showed no abnormality based on World Small Animal Veterinary Association guidelines [5].

\section{Immunoblot analysis}

Immunoblot analysis was performed as previously described with some modification [14]. One biopsy sample of the colonic mucosa for each dog was homogenized with a plastic pestle in lysis buffer ( $50 \mu \mathrm{l}$ per $1 \mathrm{mg}$ of biopsy sample) containing $50 \mathrm{mM}$ Tris- $\mathrm{HCl}$ (pH, 7.4), 1 mM EDTA, 2\% SDS and a protease inhibitor cocktail (Complete Mini EDTA-free, Roche Diagnostics $\mathrm{GmbH}$, Mannheim, Germany). Then, lysates were incubated for $30 \mathrm{~min}$ on ice and passed through a biopolymer-shredding system (QIAshredder, QIAGEN, Hilden, Germany). The protein concentration was determined by the Lowry method with a protein assay kit (Bio-Rad DC Protein Assay, Bio-Rad Laboratories Inc., Hercules, CA, U.S.A.) that included bovine serum ALB as a standard. Aliquots (approx $20 \mu \mathrm{g}$ ) of proteins were separated with PAGE and transferred to a polyvinylidene difluoride filter (Trans-Blot Turbo Mini PVDF Transfer Pack, Bio-Rad Laboratories Inc.), followed by blocking with 5\% nonfat milk, incubation with rabbit polyclonal antibodies against claudin-1, -2, -3, -5, -7 and -8 (polyclonal antibody designations: JAY.8, MH44, Z23.JM, Z43.JK, ZMD.241 and ZMD.446, respectively; Zymed Laboratories, Carlsbad, CA, U.S.A.; final concentration, $0.08 \mu \mathrm{g} / \mathrm{m} l \mathrm{diluted}$ in $5 \%$ nonfat milk), a mouse monoclonal antibody against claudin-4 (clone 3E2C1; Zymed Laboratories; $0.17 \mu \mathrm{g} / \mathrm{ml}$ ), mouse monoclonal antibody against E-cadherin (clone 36/E-cadherin; BD Biosciences, San Jose, CA, U.S.A.; $0.04 \mu \mathrm{g} / \mathrm{m} l$ ), mouse monoclonal antibody against $\beta$-catenin (clone 14/Beta-catenin; BD Biosciences; $0.06 \mu \mathrm{g} / \mathrm{m} l$ ) or mouse monoclonal antibody against $\beta$-actin (clone AC- 74; Sigma-Aldrich, St. Louis, MO, U.S.A.; $0.02 \mu \mathrm{g} / \mathrm{ml}$ ) for $2 \mathrm{hr}$. Membranes were then incubated with horseradish peroxidase-conjugated swine anti-rabbit IgG polyclonal antibody (DakoCytomation, Tokyo, Japan; $0.11 \mu \mathrm{g} / \mathrm{m} l$ ) or goat anti-mouse IgG polyclonal antibody (Rockland, Gilbertsville, PA, U.S.A.; $0.5 \mu \mathrm{g} / \mathrm{m} l$ ) for $1 \mathrm{hr}$. Signals were detected with a chemiluminescent detection reagent (ECL Prime Western Blotting Detection Reagent, GE Healthcare, Buckinghamshire, U.K.). Densitometric analyses of immunoblot images were performed with software (LumiVision Analyzer 2.0, Aisin Seiki Co., Kariya, Japan). Canine renal cortex and medulla were used as positive control tissue for claudin-1 and -8 as previously reported [14]. The intensity of the signals of each TJ and $\mathrm{AJ}$ protein was normalized to the corresponding $\beta$-actin signal.

\section{Immunohistochemical analysis}

Immunohistochemical analysis was conducted on 3- $\mu$ m-thick paraffin embedded sections of colorectal samples obtained from 5 MDs with ICRPs and 5 healthy beagles. Heat-induced antigen retrieval was performed by autoclaving the sections for 15 min at $105^{\circ} \mathrm{C}$ in buffer solution for epitope retrieval (Target Retrieval Solution, pH 9, DakoCytomation). Endogenous peroxidase activity was blocked by incubation with $\mathrm{H}_{2} \mathrm{O}_{2}$ solutions at room temperature for $30 \mathrm{~min}$. Sections were blocked with $10 \%$ goat- or rabbitserum (Histofine SAB-PO Kit, Nichirei Biosciences Inc., Tokyo, Japan) for $30 \mathrm{~min}$ at room temperature. The primary antibodies against claudin-2, $-3,-5$ and $-7(1.2 \mu \mathrm{g} / \mathrm{m} l)$ and monoclonal antibodies against claudin-4 $(1.2 \mu \mathrm{g} / \mathrm{m} l)$, E-cadherin and $\beta$-catenin $(0.8 \mu \mathrm{g} / \mathrm{m} l)$ were added, and the slides were incubated overnight at $4^{\circ} \mathrm{C}$. Negative controls were incubated with control rabbit and mouse IgG (1.2 and $0.8 \mu \mathrm{g} / \mathrm{ml}$, respectively). The slides were washed 3 times with PBS and incubated with biotinylated secondary antibody (Histofine SAB-PO Kit, Nichirei Biosciences Inc.) for $1 \mathrm{hr}$ and then rinsed. To detect the complex formed by the primary and secondary antibodies, slides were incubated with streptavidin (Histofine SAB-PO Kit, Nichirei Biosciences Inc.) for 30 min and rinsed in PBS for $10 \mathrm{~min}$. Thereafter, 3,3'-diaminobenzidine was added to the slides for $5 \mathrm{~min}$, followed by washing in water, counterstaining with Mayer's hematoxylin, dehydration and mounting with aqueous permanent medium. Images were obtained with a microscope equipped with a camera (BZ-X710, Keyence, Osaka, Japan). Stained tissue sections were evaluated at $\times 400$ magnification. Immunostaining of TJ and AJ proteins was evaluated along the length of colonic crypt base to luminal surface in areas of intact luminal epithelium. Stain intensity was subjectively graded as absent $(-)$ or positive $(+)$ based on staining results of negative control IgG. For evaluation, colonic epithelium was divided into luminal surface and crypt regions.

\section{Statistics}

Statistical analyses were performed using a computer program (JMP 11, SAS Institute Inc., Cary, NC, U.S.A.). The normality of 


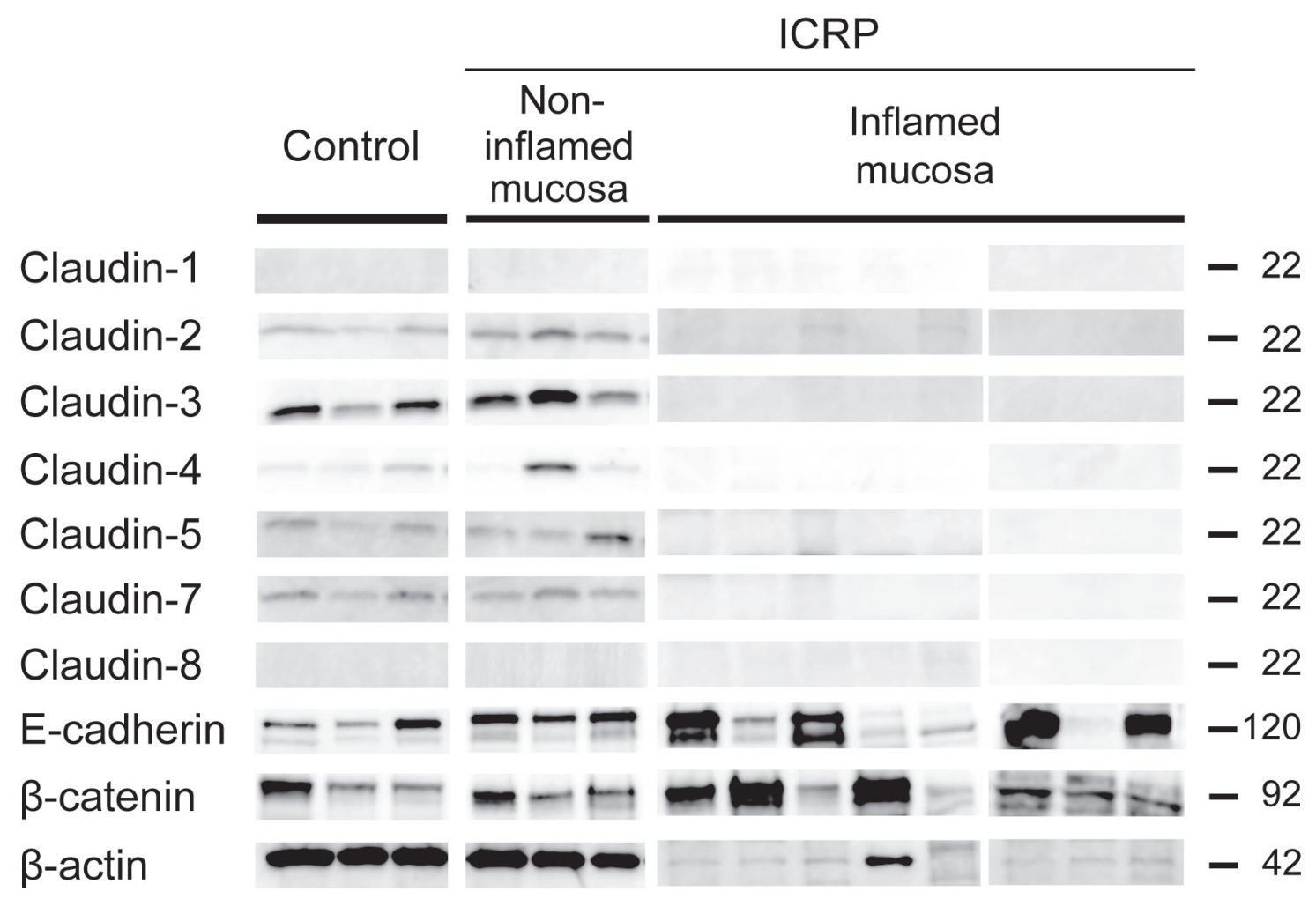

Fig. 2. Photographs of immunoblots that indicate expressions of various tight junction and adherence junction proteins in colorectal mucosa biopsy samples obtained from control dogs, and non-inflamed mucosa and inflamed mucosa of MDs with ICRPs. The photograph of control dogs and non-inflamed mucosa of MDs with ICRPs shows the representative immunoblots of each group. The photograph of inflamed mucosa of MDs with ICRPs shows the results of all of the 8 dogs. $\beta$-actin is included as a loading control. Values on the right side indicate the apparent molecular mass (in kilodaltons) of each protein.

the distribution was assessed with the Shapiro-Wilk W test; some data were determined to be non-normally distributed. Expression levels of each protein were compared between control dogs and MDs with ICRPs using the Mann-Whitney $U$ test. Values of $P<0.05$ were considered significant.

\section{RESULTS}

\section{TJ and AJ protein expression in colorectal mucosa samples}

In colonic mucosa from control dogs and non-inflamed mucosa of MDs with ICRPs, expressions of claudin-2, -3, -4, -5 and -7; E-cadherin; and $\beta$-catenin were observed (Fig. 2). Expressions of claudin-1 and -8 were not detected in colorectal mucosa samples in any dogs, although anti-claudin-1 and -8 antibodies used in this study detected these claudins in the renal cortex and medulla of dog kidney (Supplementary Fig. 1). In the inflamed mucosa of ICRPs, expressions of E-cadherin and $\beta$-catenin were observed. On the other hand, no expressions of any claudins were detected. In addition, $\beta$-actin was only slightly and heterogeneously detected. Thus, densitometry analysis of the expression of TJ and AJ proteins could not be performed in the inflamed mucosa of ICRPs. Densitometry analysis of the expression of TJ and AJ proteins in colonic mucosa from control dogs and non-inflamed mucosa of MDs with ICRPs revealed that there were no significant differences in expression levels of claudin-2, $-3,-4,-5$ or -7 , E-cadherin or $\beta$-catenin between MDs with ICRPs and control dogs (Fig. 3).

\section{Immunostaining of TJ and AJ proteins in colorectal mucosa samples}

Next, we examined the TJ and AJ proteins expression and distribution by immunohistochemistry using the paraffin-embedded tissues of surgically excised inflamed lesions that consisted of sequential histological changes from stage I to stage II lesions. Immunostaining was performed for the $\mathrm{TJ}$ and $\mathrm{AJ}$ proteins detected by immunoblot analysis. In control dogs, the expressions of claudin-3, $-4,-5$ and -7 , E-cadherin and $\beta$-catenin were observed in the colonic epithelium and seemed to be uniform along the crypt-to-luminal surface axis (Figs. 4 and 5). Claudin-2 was readily detectable only in the colonic epithelium of the crypt base, decreasing in intensity from the distal to proximal crypt and becoming barely detectable at the luminal surface of the colon. TJ and AJ proteins specific labeling was detected in the intestinal epithelium, but not in the lamina propria. In the non-inflamed mucosa of MDs with ICRPs, the expression and distribution of TJ and AJ proteins were the same as in colonic mucosa of control dogs (data not shown). In the inflamed lesions of MDs with ICRPs, similar to the results of control dogs, expressions of claudin-2, -3 , 

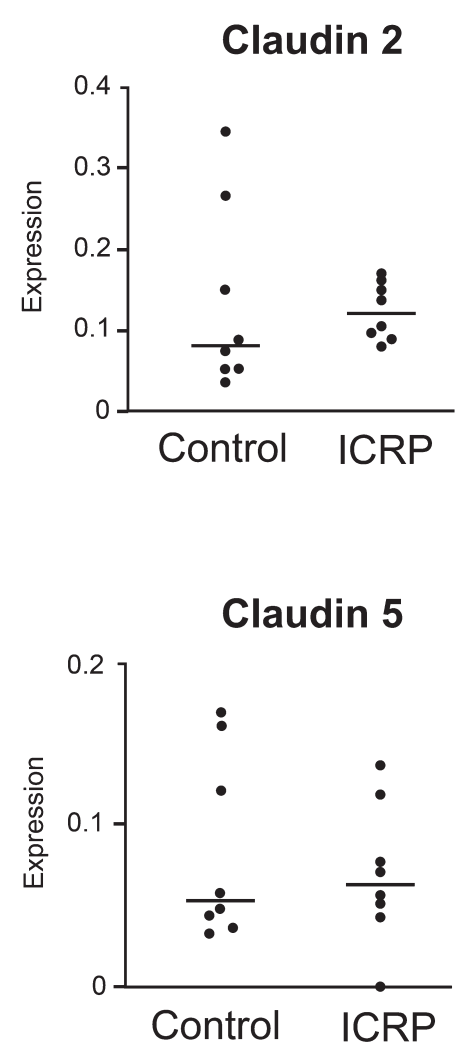
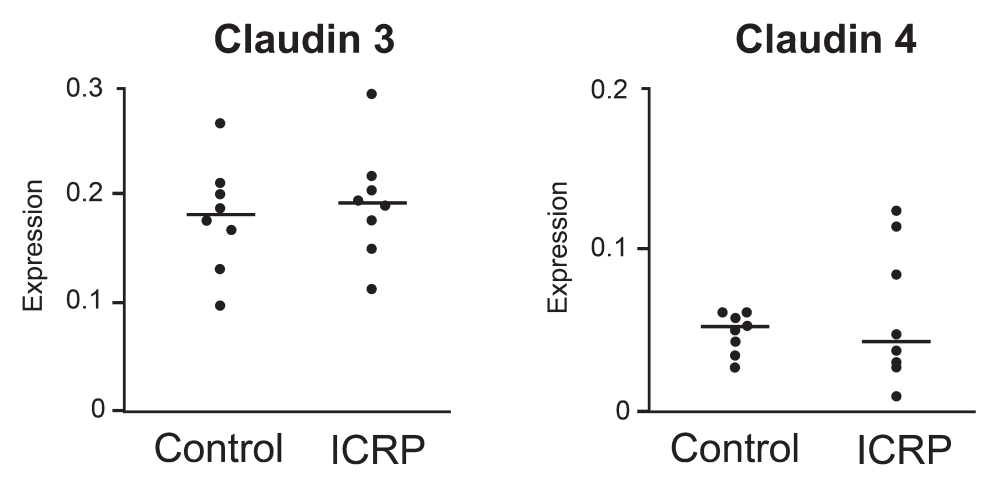

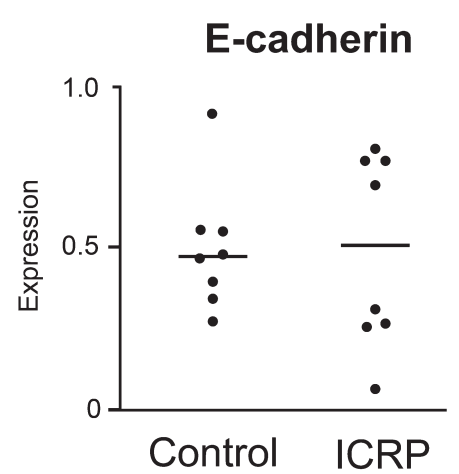

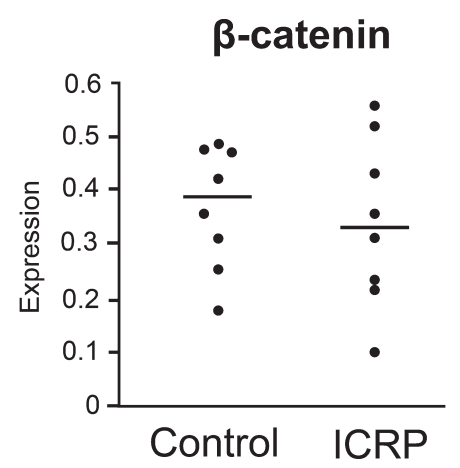

Fig. 3. Scatterplots of the expressions of claudin-2, $-3,-4,-5$ and -7 and E-cadherin; $\beta$-catenin in colorectal mucosa samples of control dogs ( $n=8$ ) and non-inflamed mucosa of MDs with ICRPs $(n=8)$. The horizontal line for each group represents the median. Expression units for each protein relative to $\beta$-actin expression.

$-4,-5$ and -7 , E-cadherin and $\beta$-catenin were detected in the colonic epithelium within stages I and II (Figs. 4 and 5). Meanwhile, the expression of TJ and AJ proteins was almost abolished in granulation tissue (stage III), because of a limited amount of the remaining epithelial component (data not shown). Negative control staining with rabbit and mouse IgG incubation showed no positive reaction in the colorectal samples of control dogs and MDs with ICRPs (Supplementary Fig. 2).

\section{DISCUSSION}

In the present study, we examined the expression and distribution of $\mathrm{TJ}$ and $\mathrm{AJ}$ proteins in the colorectal mucosa of MDs with ICRPs. The results demonstrated that no alteration in the expression and distribution of TJ and AJ proteins was observed in the colorectal epithelium within the inflamed lesions of MDs with ICRPs.

By immunoblotting, claudin-2, -3, -4, -5 and -7 expressions were detected in the non-inflamed mucosa of MDs with ICRPs and colonic mucosa of control dogs. In addition, there were no significant differences in expression levels of claudin-2, $-3,-4,-5$ or -7 between the non-inflamed mucosa of MDs with ICRPs and those of control dogs. In contrast, all claudin protein expressions 

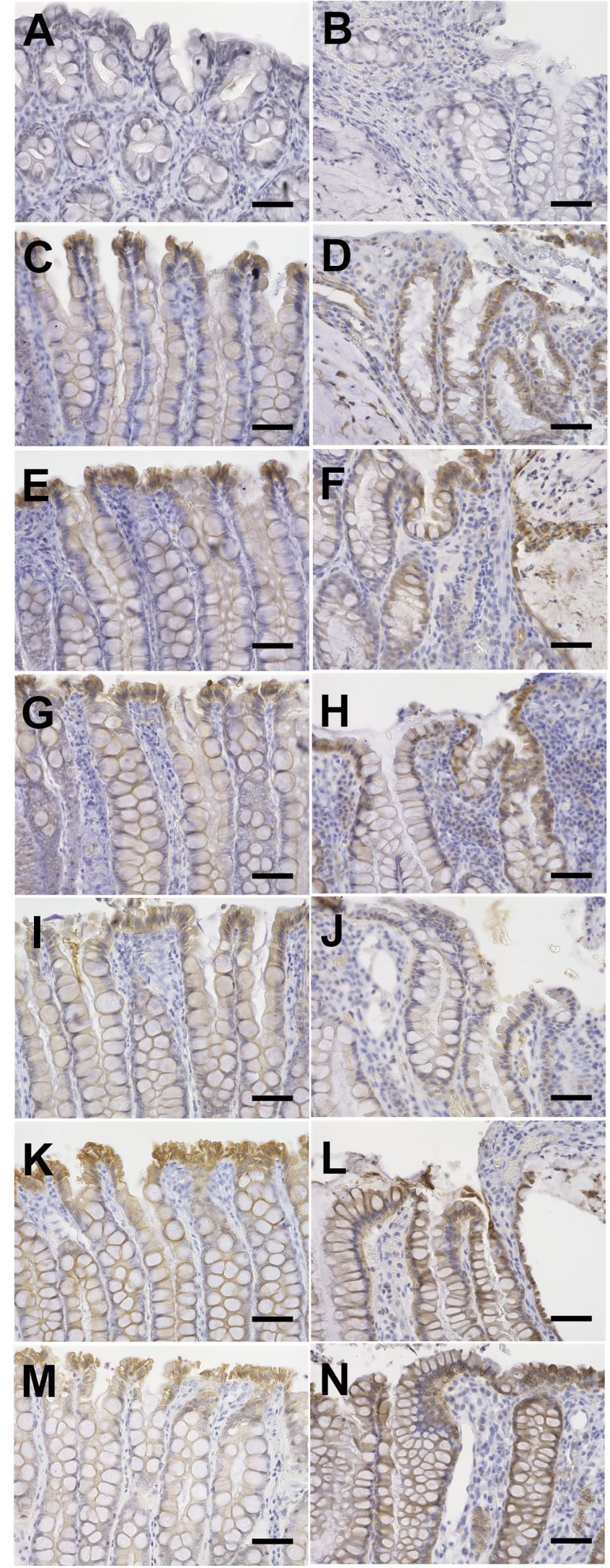

Fig. 4. Photomicrographs of representative immunostaining results of claudin-2 (A and B), -3 (C and D), $-4(\mathrm{E}$ and $\mathrm{F}),-5(\mathrm{G}$ and $\mathrm{H})$ and -7 (I and $\mathrm{J}$ ) and E-cadherin (K and $\mathrm{L}$ ); $\beta$-catenin ( $\mathrm{M}$ and $\mathrm{N}$ ) on the luminal surface of colonic mucosa from control dogs (left column) and stage I to stage II inflamed mucosa (right column) of ICRPs. Bars $=50 \mu \mathrm{m}$.
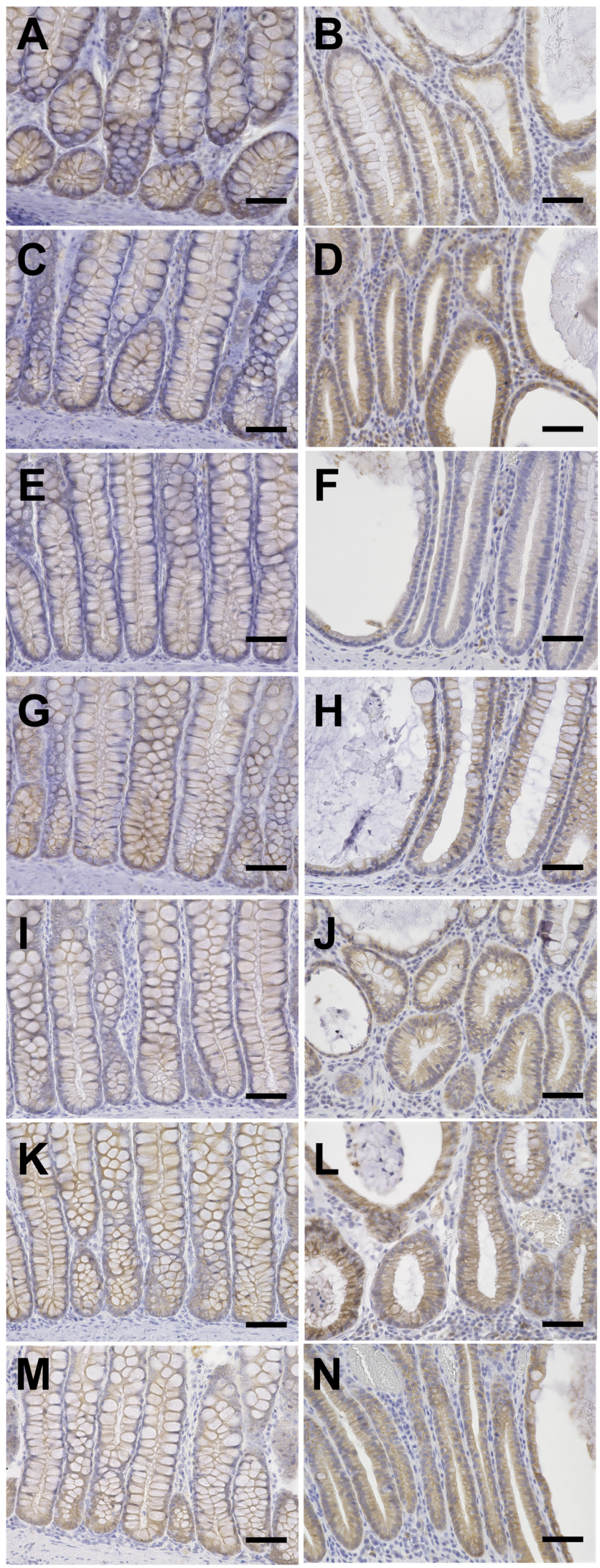

Fig. 5. Photomicrographs of representative immunostaining results of claudin-2 (A and B), -3 (C and D), -4 (E and F), -5 ( $\mathrm{G}$ and $\mathrm{H})$ and -7 (I and $\mathrm{J}$ ), and $\mathrm{E}$-cadherin ( $\mathrm{K}$ and $\mathrm{L}) ; \beta$-catenin $(\mathrm{M}$ and $\mathrm{N})$ in the crypt base of colonic mucosa from control dogs (left column) and stage I to stage II inflamed mucosa (right column) of ICRPs. Bars $=50 \mu \mathrm{m}$. 
were undetectable in the inflamed lesions of ICRPs. A possible reason for these results is that we obtained endoscopic biopsy samples from the center of inflamed lesions, which was mainly composed of granulation tissue, and a scant amount of the epithelial component remained. Basically, claudin proteins are expressed on the epithelium in the intestinal mucosa [1, 15]. Thus, undetectable levels of expression of claudin proteins in the inflamed lesion might simply reflect a reduced amount of the epithelial component. It is difficult to macroscopically differentiate stage I and II lesions from stage III lesions during the endoscopic procedure. Thus, it was thought that immunoblotting was not a suitable method for the analysis of claudin protein expressions in the inflamed mucosa, because of a large amount of granulation tissue in the inflamed lesion, especially in stage III.

In contrast, E-cadherin and $\beta$-catenin proteins were detected in inflamed lesions of ICRP. The reason for detectable levels of E-cadherin and $\beta$-catenin proteins, but not claudin protein, is unknown, but it may be due to the high sensitivity of antibodies for these proteins used in this study. These antibodies could detect E-cadherin and $\beta$-catenin proteins on the scant amount of remaining epithelium in the inflamed lesion. However, $\beta$-actin expression was barely detectable in the inflamed lesions of ICRPs. Thus, densitometric analysis of E-cadherin and $\beta$-catenin protein expressions could not be performed. It was also unclear why $\beta$-actin expression was barely detectable. A possible explanation for this result was that the biopsy samples from inflamed mucosa might not contain sufficient amount of cells for detecting $\beta$-actin expression, although the fixed amount of proteins (approx $20 \mu \mathrm{g}$ ) were used for immunoblot analysis. The inflamed mucosa of ICRPs was composed of heterogeneous tissue architecture, including dilated crypts with abundant mucus material and inflammatory cell debris as shown in Fig. 1. Indeed, $\beta$-actin protein showed heterogeneous expression in the inflamed mucosa of ICRPs as shown in Fig. 2. Moreover, we also used anti-glyceraldehyde 3-phosphate dehydrogenase monoclonal antibody as an internal control for protein expression, but the result was the same as that of the anti- $\beta$-actin antibody (data not shown). From these results, it is thought to be difficult to examine the TJ and AJ proteins expression in the inflamed mucosa of ICRPs by immunoblotting.

To further compare the expressions of the TJ and AJ proteins between the inflamed lesions of ICRPs and colorectal mucosa of control dogs, we employed immunohistochemical analysis, which enabled us to examine the TJ and AJ protein expressions on the epithelium of stage I and stage II lesions of ICRPs. As a result, there was no alteration in the expression and distribution of TJ and AJ proteins even in the epithelium of the stage II lesions of ICRPs. These findings suggest that significant changes in apical junction complex proteins expression might not occur in the colorectal epithelium of ICRPs. In human IBD, barrier integrity of the intestinal mucosa was evaluated by not only TJ and AJ proteins expression analysis, but also the assessment of barrier functions $[12,23]$. In the future study, evaluation of the barrier function, such as transepithelial electrical resistance and flux assays of molecular tracers, in the colorectal mucosa of MDs with ICRPs is needed.

On the other hand, in human IBD, several previous studies reported that claudin-2 protein expression was upregulated in the colonic epithelium of affected lesions [21,23]. Furthermore, it has been reported that alterations of claudin-1, $-3,-4,-7$ and -8 expressions were observed in the colonic mucosa of human IBD [1,21]. In human IBD, the alterations in expressions of claudins were reported to be induced by pro-inflammatory cytokines (IFN- $\gamma$, TNF- $\beta$ and IL-6) and Th2 cytokine (IL-13) [7, 17, 23]. In MDs with ICRPs, similar to human IBD, IFN- $\gamma$ and TNF- $\beta$ were upregulated in inflamed mucosa $[16,18]$. However, the present study demonstrated no alteration in the expression or distribution of several TJ proteins in MDs with ICRPs. There is a possibility of changes in the expression of the other TJ proteins, such as occludin and Zona occludens-1 [10], in inflamed lesions of MDs with ICRPs.

There are several limitations in the current study. Firstly, the sample size of each group was small. The possibility of alterations in $\mathrm{TJ}$ and $\mathrm{AJ}$ protein expressions could not be definitely ruled out, because of the small number of cases. Secondly, this study examined only seven claudin proteins and two AJ proteins. However, the TJ and AJ are composed of a number of transmembrane proteins, including occludin, junctional adhesion molecule, tricelluin, $\mathrm{p}$-cadherin and $\alpha$-catenin which might play important roles in forming the TJ and AJ $[12,19]$. A further study is needed to examine the expressions of the other TJ and AJ proteins in inflamed lesions of MDs with ICRPs.

In conclusion, the results of the present study indicate that there was no clear change in expressions of several TJ and AJ proteins in the colorectal epithelium of ICRPs. A further study is needed to examine the other TJ and AJ proteins expression and to evaluate the barrier function of colorectal mucosal epithelium from MDs with ICRPs.

\section{REFERENCES}

1. Barmeyer, C., Schulzke, J. D. and Fromm, M. 2015. Claudin-related intestinal diseases. Semin. Cell Dev. Biol. 42: 30-38. [Medline] [CrossRef]

2. Capaldo, C. T. and Nusrat, A. 2015. Claudin switching: Physiological plasticity of the Tight Junction. Semin. Cell Dev. Biol. 42: 22-29. [Medline] [CrossRef]

3. Cereijido, M., Shoshani, L. and Contreras, R. G. 2000. Molecular physiology and pathophysiology of tight junctions. I. Biogenesis of tight junctions and epithelial polarity. Am. J. Physiol. Gastrointest. Liver Physiol. 279: G477-G482. [Medline]

4. Cerquetella, M., Spaterna, A., Laus, F., Tesei, B., Rossi, G., Antonelli, E., Villanacci, V. and Bassotti, G. 2010. Inflammatory bowel disease in the dog: differences and similarities with humans. World J. Gastroenterol. 16: 1050-1056. [Medline] [CrossRef]

5. Day, M. J., Bilzer, T., Mansell, J., Wilcock, B., Hall, E. J., Jergens, A., Minami, T., Willard, M., Washabau R., World Small Animal Veterinary Association Gastrointestinal Standardization Group. 2008. Histopathological standards for the diagnosis of gastrointestinal inflammation in endoscopic biopsy samples from the dog and cat: a report from the World Small Animal Veterinary Association Gastrointestinal Standardization Group. J. Comp. Pathol. 138 Suppl 1: S1-S43. [Medline] [CrossRef]

6. Fukushima, K., Eguchi, N., Ohno, K., Kanemoto, H., Takahashi, M., Igarashi, H., Ohmi, A., Nakashima, K. and Tsujimoto, H. 2016. Efficacy of leflunomide for treatment of refractory inflammatory colorectal polyps in 15 Miniature Dachshunds. J. Vet. Med. Sci. 78: 265-269. [Medline] 
[CrossRef]

7. Heller, F., Florian, P., Bojarski, C., Richter, J., Christ, M., Hillenbrand, B., Mankertz, J., Gitter, A. H., Bürgel, N., Fromm, M., Zeitz, M., Fuss, I., Strober, W. and Schulzke, J. D. 2005. Interleukin-13 is the key effector Th2 cytokine in ulcerative colitis that affects epithelial tight junctions, apoptosis, and cell restitution. Gastroenterology 129: 550-564. [Medline] [CrossRef]

8. Igarashi, H., Ohno, K., Fujiwara-Igarashi, A., Kanemoto, H., Fukushima, K., Uchida, K. and Tsujimoto, H. 2015. Inflammatory colorectal polyps in miniature Dachshunds frequently develop ventrally in the colorectal mucosa. Vet. J. 203: 256-258. [Medline] [CrossRef]

9. Igarashi, H., Ohno, K., Horigome, A., Fujiwara-Igarashi, A., Kanemoto, H., Fukushima, K., Odamaki, T. and Tsujimoto, H. 2016. Fecal dysbiosis in miniature dachshunds with inflammatory colorectal polyps. Res. Vet. Sci. 105: 41-46. [Medline] [CrossRef]

10. Landy, J., Ronde, E., English, N., Clark, S. K., Hart, A. L., Knight, S. C., Ciclitira, P. J. and Al-Hassi, H. O. 2016. Tight junctions in inflammatory bowel diseases and inflammatory bowel disease associated colorectal cancer. World J. Gastroenterol. 22: 3117-3126. [Medline] [CrossRef]

11. Laukoetter, M. G., Nava, P. and Nusrat, A. 2008. Role of the intestinal barrier in inflammatory bowel disease. World J. Gastroenterol. 14: 401-407. [Medline]

12. Mehta, S., Nijhuis, A., Kumagai, T., Lindsay, J. and Silver, A. 2015. Defects in the adherens junction complex (E-cadherin/ $\beta$-catenin) in inflammatory bowel disease. Cell Tissue Res. 360: 749-760. [Medline] [CrossRef]

13. Ohmi, A., Tsukamoto, A., Ohno, K., Uchida, K., Nishimura, R., Fukushima, K., Takahashi, M., Nakashima, K., Fujino, Y. and Tsujimoto, H. 2012. A retrospective study of inflammatory colorectal polyps in miniature dachshunds. J. Vet. Med. Sci. 74: 59-64. [Medline] [CrossRef]

14. Ohta, H., Yamaguchi, T., Rajapakshage, B. K., Murakami, M., Sasaki, N., Nakamura, K., Hwang, S. J., Yamasaki, M. and Takiguchi, M. 2011. Expression and subcellular localization of apical junction proteins in canine duodenal and colonic mucosa. Am. J. Vet. Res. 72: $1046-1051$. [Medline] [CrossRef]

15. Ohta, H., Sunden, Y., Yokoyama, N., Osuga, T., Lim, S. Y., Tamura, Y., Morishita, K., Nakamura, K., Yamasaki, M. and Takiguchi, M. 2014. Expression of apical junction complex proteins in duodenal mucosa of dogs with inflammatory bowel disease. Am. J. Vet. Res. 75: 746-751. [Medline] [CrossRef]

16. Ohta, H., Takada, K., Torisu, S., Yuki, M., Tamura, Y., Yokoyama, N., Osuga, T., Lim, S. Y., Murakami, M., Sasaki, N., Nakamura, K., Yamasaki, M. and Takiguchi, M. 2013. Expression of CD4+ T cell cytokine genes in the colorectal mucosa of inflammatory colorectal polyps in miniature dachshunds. Vet. Immunol. Immunopathol. 155: 259-263. [Medline] [CrossRef]

17. Suzuki, T., Yoshinaga, N. and Tanabe, S. 2011. Interleukin-6 (IL-6) regulates claudin-2 expression and tight junction permeability in intestinal epithelium. J. Biol. Chem. 286: 31263-31271. [Medline] [CrossRef]

18. Tamura, Y., Ohta, H., Torisu, S., Yuki, M., Yokoyama, N., Murakami, M., Lim, S. Y., Osuga, T., Morishita, K., Nakamura, K., Yamasaki, M. and Takiguchi, M. 2013. Markedly increased expression of interleukin-8 in the colorectal mucosa of inflammatory colorectal polyps in miniature dachshunds. Vet. Immunol. Immunopathol. 156: 32-42. [Medline] [CrossRef]

19. Tsukita, S., Furuse, M. and Itoh, M. 2001. Multifunctional strands in tight junctions. Nat. Rev. Mol. Cell Biol. 2: 285-293. [Medline] [CrossRef]

20. Uchida, E., Chambers, J. K., Nakashima, K., Saito, T., Ohno, K., Tsujimoto, H., Nakayama, H. and Uchida, K. 2016. Pathologic Features of Colorectal Inflammatory Polyps in Miniature Dachshunds. Vet. Pathol. 53: 833-839. [Medline] [CrossRef]

21. Weber, C. R., Nalle, S. C., Tretiakova, M., Rubin, D. T. and Turner, J. R. 2008. Claudin-1 and claudin-2 expression is elevated in inflammatory bowel disease and may contribute to early neoplastic transformation. Lab. Invest. 88: 1110-1120. [Medline] [CrossRef]

22. Xavier, R. J. and Podolsky, D. K. 2007. Unravelling the pathogenesis of inflammatory bowel disease. Nature 448: 427-434. [Medline] [CrossRef]

23. Zeissig, S., Bürgel, N., Günzel, D., Richter, J., Mankertz, J., Wahnschaffe, U., Kroesen, A. J., Zeitz, M., Fromm, M. and Schulzke, J. D. 2007. Changes in expression and distribution of claudin 2, 5 and 8 lead to discontinuous tight junctions and barrier dysfunction in active Crohn's disease. Gut 56: 61-72. [Medline] [CrossRef] 